\title{
2 Ziel der Arbeit
}

Ziel der Arbeit ist es, anhand der Auswertung von theoretischen Kenntnissen und Meinungen und der darauf aufbauenden analytischen Forschung das Risiko, das sich in Summe durch die Vernetzung in der Produktion ergibt, zu bewerten und transparent zu machen. Transparent bedeutet in diesem Fall, dass die Möglichkeit geschaffen wird, konkrete technische Risiken so zu aggregieren, dass die potenziellen Auswirkungen auch auf der Ebene der Geschäftsberichte ersichtlich werden. Die Risiken durch die Vernetzung in der Produktion sollen zumindest auf der Ebene der Geschäftsberichte auch als finanzielle Risiken dargestellt werden. Damit einher geht das Ziel, operative Risiken finanziell greifbar zu machen, also eine Quantifizierung zu bewirken. Im Idealfall bietet die entwickelte Bewertungsmethode die Möglichkeit, individuelle Anpassungen vornehmen zu können. Um das Ziel der Anpassungsfähigkeit verfolgen zu können, ist es unausweichlich, sowohl die Entwicklung als auch die Methode selbst absolut transparent zu gestalten. Dies bedeutet auch, möglichst stark an den aktuellen Stand der Forschung anzuknüpfen, um zu einer Lösung des Problems zu kommen. 